Chapter 16

\title{
The Neurofunctional Architecture of Motor Imagery
}

\author{
Aymeric Guillot, Franck Di Rienzo and \\ Christian Collet \\ Additional information is available at the end of the chapter \\ http://dx.doi.org/10.5772/30961
}

\section{Introduction}

The remarkable ability of the human brain to mentally simulate sensations and actions has become a major focus of research during the last three decades. Such mental simulation process is called mental imagery and resembles perception, but without the immediate actual corresponding sensory input. Typically, mental imagery is often referred to as the experience of seeing with the mind's eye, hearing with the mind's ear. In other words, mental images could be generated from any sensorial system. Imagery experience is usually understood as evocation, copy, or reconstruction of actual perceptual experience from the long term memory systems, which is first recalled and then internally reproduced within working memory. At other times, participants may also anticipate possible or forthcoming events through imagery as a consequence of what they are expecting. A key feature of this simulation process is that it gives the ability to mentally rehearse motor acts without overt body movement, which is known as the motor imagery (MI) experience. Practically, MI is more often used when the whole human body or body segments are involved, hence requiring participants to imagine the body as the generator of acting forces and not only the effects of these forces on the external world. In this latter case, the information from the body is mainly used to build the mental image of the movement, e.g. tactile or any kind of proprioceptive data, from the muscles, the joints or the inner ear. In the wealth of motor learning literature, it is now well-established that MI is a valuable complement to physical practice to enhance cognitive and motor performance(Driskell et al., 1994; Guillot \& Collet, 2008), as well as to promote motor recovery (Sharma, Pomeroy, \& Baron, 2006; de Vries \& Mulder, 2007; Di Rienzo et al., 2014a). Interestingly, different types of MI can be described and easily combined. While people commonly report using visual imagery (either through a first-person or third-person perspective), auditory imagery, and kinaesthetic imagery (Kosslyn et al., 1990), each perceptual modality can be accompanied by imagery. On the other hand, and with reference to perception, mental imagery combines several cues from 
different sensorial systems, thus making the imagery a global sensory-motor experience. Thus, investigating and assessing MI remains difficult due to its concealed nature.

Early on, MI has attracted attention from cognitive neuroscientists. In particular, understanding the neural correlates of goal-directed action, whether executed or imagined, has been an important purpose of cognitive brain research since the advent of neuroimaging techniques such as functional Magnetic Resonance Imaging (fMRI). Providing physiological recordings that correlate to the MI experience was therefore a major issue of the past two decades. In short, but not only, neuroimaging research demonstrated that MI engages motor systems, and that the cerebral plasticity due to actual practice also occurred during MI as well. These findings help to explain why MI can improve actual performance, and further contribute to motor memory consolidation. The present chapter was designed to provide an overview of the neural correlates of MI, with a threefold aim: $i$ ) to explain why recording human brain functioning with fMRI during MI is of particular interest and provides an objective measurement of imagery processes, ii) to give a clear picture of the neural networks mediating MI and actual execution of the same movement, while considering the issue of brain plasticity in the light of individual differences in imagery ability, motor expertise, the different forms of imagery, and the level of motor learning, and iii) to consider the recent fMRI methodological advances, e.g. using real-time fMRI, as well as recording changes in functional and effective connectivity in the neural networks activated during MI.

\section{Using fMRI to assess brain activity during motor imagery}

The most common aim of fMRI data analysis is to investigate the neural processes mediating higher cognitive functions, and detect correlations between brain activations and the task the participants have to perform during the scan. Since the early 1980s, fMRI has come to dominate the MI-related brain mapping research. During typical fMRI image acquisitions, participants are asked to alternatively perform a movement, either physically or mentally. These experimental conditions are also compared with a rest/control condition.

There are two main types of experimental design in fMRI: the block design and the eventrelated design. The block design paradigm consists of several epochs representing experimental conditions, during which stimuli are presented and actual execution or MI are required from participants, which are interleaved with time blocks of rest. Event-related designs rather associate brain processes with discrete events, with stimulus events being randomly presented one at a time, and being separated by an inter-stimulus interval of a pre-determined length. Habituation effects can be reduced, and stimuli are usually presented either tens of seconds apart or at a faster rate (e.g., every second). It should be noted that event-related designs are more sensitive to the details of the hemodynamic response model use. Figure 1 provides a schematic illustration of these two experimental designs when comparing the neural networks mediating MI and actual execution of the same movement.

There is not a universal procedure yet to acquire and process functional data and many researchers independently developed their methods and analyses, using different software. 


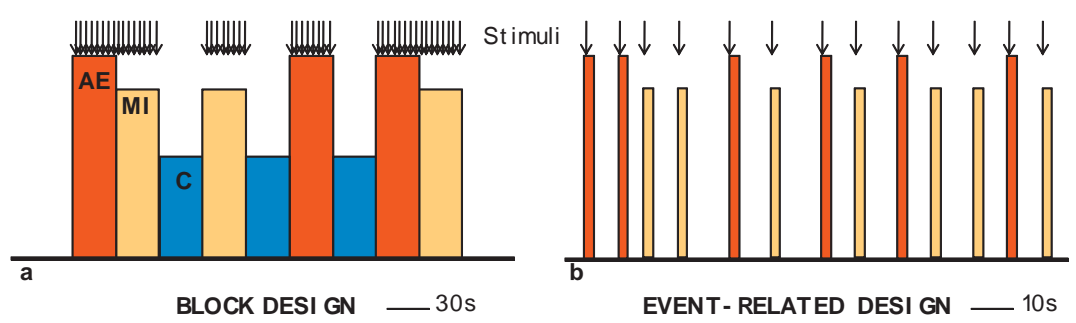

Figure 1. Schematic representation of a block design (a) and an event-related design (b) fMRI paradigm. In the block design paradigm, sustained periods of 20-30 s are used for actual execution (AE), motor imagery (MI) or control (C) conditions during which several trials are performed successively (vertical arrows). Before each block of practice, brief periods of time (2-3s) are necessary to provide participants some instructions related to the forthcoming condition. Brief 'rest' periods can also be included. In the event-related design, each experimental condition is presented at random (orange and red vertical bars and vertical arrows).

Practically, the most commonly technique used to map brain activity is based on the Blood Oxygenation Level Dependent (BOLD) effect, measuring regional differences in hemoglobin oxygenation. Therefore, a considerable amount of experimental studies investigated BOLD changes during MI. They primarily aimed at comparing the neuronal activations recorded during MI and actual practice of the same movement. For example, Grèzes and Decety (2001) provided evidence of a functional equivalence between intending, simulating, observing, and performing an action, as shown by the great deal of overlap among the corresponding neural substrates. Interestingly, brain activations recorded during MI do however not exactly match those of actual movement execution (Lotze \& Halsband, 2006). Some differences also appear when comparing different types of MI or with regard to the individual features of the sample of participants. Put simply, fMRI allows the measurement of different neuronal activity in participants with high vs. low MI ability (Guillot et al., 2008), as well as differences in the patterns of cerebral activity related to the degree of expertise of the participants with the motor task (Lotze et al., 2003; Ross et al., 2003; Milton et al., 2007, 2008). Based on such evidence, fMRI offers a way to evidence the MI experience (Guillot et al., 2008). Finally, recent fMRI data suggested that different types of imagery elicit different patterns of neural activation (Solodkin et al., 2004; Jackson et al., 2006; Guillot et al., 2009; Lorey et al., 2009), while others nicely demonstrated, through positron emission tomography (PET), that the cerebral plasticity occurring after physical practice was reflected during MI (Lafleur et al., 2002; Jackson et al., 2003), hence reinforcing the principle of functional equivalence between MI and physical performance of the same task. Before we consider these issues and review the main related findings in the next section, an important methodological question remains to be addressed. Specifically, what are the additional benefits provided by fMRI to the study of MI compared to other neuroimaging and electrophysiological techniques, and, in turn, what are the limitations of fMRI recordings?

A first advantage of fMRI, compared to PET, is its non-invasive nature. There is no radiation exposure, and no need for injection of radioactive materials with fMRI. Another strength is its high spatial resolution allowing the measurement of brain activity from deep structures. Unlike electrophysiological techniques such as electroencephalography (EEG) or even 
magnetoencephalography (MEG) which are somewhat biased towards the cortical surface, fMRI records signal from all regions of the brain, including subcortical structures. This is of particular importance when pinpointing the neural correlates of MI as activation of cerebellum and basal ganglia is commonly reported. As every technique, however, fMRI has also some methodological disadvantages or weaknesses for the study of MI (e.g., Dietrich, 2008). A first limit is related to the causality between brain activation and the task the participants perform in the scanner. Although the baseline activity is subtracted from that recorded during the task and the result is averaged for all participants, the causality between activated structures and characteristics of the task is not straightforward. In other words, some brain structures could remain activated although this activity is not directly related to the task. Such argument for the causality however also holds for other brain imaging techniques. Second, the temporal resolution, of about several seconds must also be considered, as fMRI cannot track the rapid temporal dynamics of the functioning brain compared to high temporal resolution methods, e.g. EEG or MEG. Neuromodulatory effects of some cognitive functions such as attention and memory are also likely to affect the spatiotemporal resolution of the signal as they can affect large masses of cells, and potentially induce larger changes in the fMRI signal than the sensory signals themselves (Logothetis, 2008). Fourth, regarding data interpretation, advanced statistical methods are required to identify active voxels, while modifications of the brain activity might be due either to an excitatory or inhibitory influence. Finally, the position in the scanner and the limitation of body movements make it difficult to investigate complex motor skills. Indeed, the participant is lying in supine position in the scanner, and then performs both actual execution and mental representation of the same movement. However, in such situation, only simple movement of fingers, hands, feet, or tongue, or more complex movements of limited amplitude can actually be studied.

As reported by Logothetis (2008), and despite the shortcomings mentioned above, fMRI remains probably the best current available technique for providing insights into brain function and formulating interesting testablehypotheses. Logothetis also stated that somelimitations of fMRI are directly due to inappropriate experimental protocols that ignore the circuitry and complex functional organization of the human brain. This is a critical aspect that must be considered before drawing general conclusions and when comparing results across experimental studies. In particular, a specific attention should be paid to the control condition from which MI-related brain activity is reported. In fMRI data analyses, images of activity from experimental and control conditions are subtracted to determine significant peaks of activation. Hence, a difference which does not reach the significant threshold does not necessarily reflect a lack of activity, but can also be due to similar brain activations during both experimental and control tasks. In other words, if the control task is not thoroughly determined, the remaining activity of some brain regions might bias the pattern of activity observed during the experimental condition, i.e. MI. Inspection of the MI literature reveals great differences across studies. Among others, participants were asked either to listen for auditory stimuli without engaging in MI (perceptual control condition), having rest, projecting into a restful state, fixating a static stimulus on a screen, or even imagining a 'neutral' static motor task. Obviously, such differences might significantly influence the pattern of activity recorded when contrasting the experimental and the control conditions. This is likely to render the functional brain map, hence hindering the conclusions that one can reach. Another example is the fact that MI can be 
performed either with open or closed eyes. Marx et al. (2003) suggested that eye closure is likely to improve imagination, but in some circumstances, MI paradigms might require participants to open their eyes to fixate a screen and/or wait for instructions. As some activation or deactivation of cortical regions could be undetected with eyes open, however, MI should ideally be performed with closed eyes as it was asked for the rest condition (Marx et al., 2004).

\section{Neural correlates of motor imagery}

MI research provided convincing evidence that there is a functional equivalence between MI and motor performance (e.g., Jeannerod, 1994; Grèzes \& Decety, 2001; Holmes \& Collins, 2001; Szameitat et al., 2012; Burianova et al., 2013; Hétu et al., 2014). It is therefore not surprising that movement execution and MI reveal a high overlap of active brain regions, i.e. imagery draws on almost the same neural network that is used in actual perception and motor control (Murphy et al., 2008). Considerable evidence in support of the functional equivalence theory comes from fMRI studies which demonstrated that both cortical and subcortical brain areas are activated during MI. All studies showed that secondary motor areas are recruited during MI, but that the primary motor cortex might also be part of the network involved in imagined actions. Cerebellar and basal ganglia activations are also consistently reported during MI (Gerardin et al., 2000; Munzert et al., 2009). Finally, apart from motor-related areas, other regions such as parietal lobules are activated during mental simulation of actions (Gao et al., 2011). Taken together, these data support that MI and motor performance share the same neural substrate (Figure 2), but interestingly there are also some differences within the pattern of activity in these areas, as well as with regards to the characteristics of the participants or the content of the MI task.

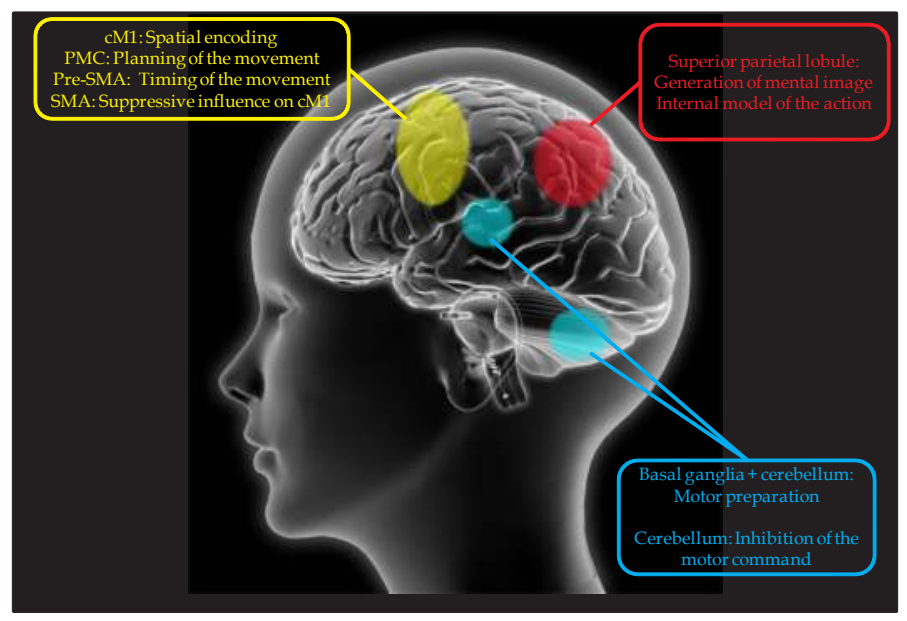

Figure 2. Schematization of brain activity during $\mathrm{MI}$ and possible roles of motor-related regions. 


\subsection{Contribution of the primary motor cortex}

Since the pioneering PET study by Roland et al. (1980), who did not find a significant activation of the contralateral primary motor cortex (cM1) during MI, the question of the contribution of cM1 during imagined actions has attracted considerable attention and remains controversial (for reviews, see Lotze \& Halsband, 2006; Sharma et al., 2008; Munzert et al., 2009; Lotze \& Zentgraf, 2010). For example, Binkofski et al. (2000), Gerardin et al. (2000), Kuhtz-Bushbeck et al. (2003) and Hanakawa et al. $(2003,2008)$ failed to find peaks of activation in cM1, while Dechent et al. (2004) reported fleeting involvement. In contrast, several studies reported significant activation in cM1 during MI (Leonardo et al., 1995; Sabbah et al., 1995; Porro et al. 1996, 2000; Roth et al., 1996; Lotze et al., 1999; Ehrsson et al. 2003; Nair et al., 2003; Solodkin et al. 2004; Guillot et al., 2008, 2009). In a seminal paper, Ehrsson et al. (2003) even showed that the content of MI was reflected in the pattern of motor cortical activation. Accordingly, they found that MI of hand, foot and tongue movements specifically activated the corresponding hand, foot and tongue sections of cM1. Transcranial magnetic studies also revealed cM1 activation during MI (for review, see Stinear et al., 2010). Finally, and as previously reviewed by Munzert et al. (2009), similar cM1 activation was found during MI of movements with the impaired limb to that during motor execution with the unimpaired limb in amputees (Ersland et al., 1996). The same result was found in healthy controls during motor execution of the same movement and in patients with spinal cord injury (Alkadhi et al., 2005; Cramer et al., 2005). The fact that these patients do not need to inhibit the movement might have influenced the nature of the MI process (Munzert et al., 2009). A recent MEG study however revealed similar patterns of activity in cM1 during MI and actual practice of the same movement in a patient with spinal cord injury, while a loss of power was observed in the matched healthy control participant (Di Rienzo et al., 2011, 2014b). These latter data support fMRI findings and further confirm both the contribution of cM1 during MI and the weakened inhibitory processes during MI in patients with spinal cord injury. Cerebral activations governing the inhibition of action during MI have not been yet fully explored. This would probably offer fruitful fMRI investigations in the future.

With regards to cM1 activation, Sharma et al. (2006) suggested that discrepancies in results may be due, at least partially, to methodological differences and difficulties in monitoring compliance with MI instructions. Definitely, the advent of fMRI techniques to investigate the contribution of cM1 during MI brought significant data into the debate. Furthermore, Sharma and colleagues argued that previous experimental studies did not specifically address whether the subdivisions of cM1 were differentially involved during MI. Furthermore, the primary motor cortex can be subdivided into an anterior component, thought to be executive in nature, while its posterior part would be involved in cognitive tasks or non-executive functions (Sanes \& Donoghue, 2000). Accordingly, Sharma et al. (2008) reported that the cluster distribution in the anterior part of cM1 was significantly reduced during MI compared to the physical execution, while that of the posterior part was similar. Accordingly, they proposed that the role of $\mathrm{cM} 1$ and its subdivisions may be related to spatial encoding. Despite these results, determining how MI participates to the activation of cM1 remains difficult. Typically, cM1 activation during $\mathrm{MI}$ is usually smaller compared to that during overt motor execution, and 
is not systematically observed in all participants (Munzert et al., 2009). It was also demonstrated that cM1 was increasingly involved along with the complexity of movements (KuhtzBuschbeck et al., 2003). As well, activation of cM1 might be differentially influenced by MI instructions, MI ability and motor expertise (Lotze \& Zentgraf, 2010).

Taken together, and despite some controversial data, fMRI studies support that cM1 is actually activated during MI but more weakly than during actual movement. Such activation is not essential for imagery and the neurons are not in the same location than those active during movement execution (Lotze \& Halsband, 2006). Moreover, cM1 might not only have an execution function for the motor system (Lotze \& Zentgraf, 2010), and it is particularly preparation for MI that may impact significantly cM1 activation (Johnson et al., 2002; Zang et al., 2003; Munzert et al., 2009).

\subsection{Activation of the other motor-related regions}

Unlike the contribution of cM1, there is a general consensus regarding the activation of secondary motor areas of the cerebral cortex during MI. Accordingly, fMRI studies provided strong evidence that motor-related areas such as the ventral and dorsal parts of the premotor cortex (PMC), the supplementary motor area (SMA) and the pre-SMA, are active during MI of both simple and complex movements as well as subcortical areas such as the cerebellum and the putamen. Overall, it was shown that MI might activate a subset of the areas required for movement execution, albeit to a lesser extent (e.g., Macuga \& Frey, 2011). Conversely, other activations could also be more robust in MI than during motor execution (e.g., Gerardin et al., 2000) thus leading to a partial overlapping of the networks mediating MI and actual execution of the same movement.

The activation of the SMA is now well-established during MI (Guillot et al., 2008; 2009; Hanakawa et al., 2003; Lotze et al., 1999; Munzert et al., 2009; Olsson et al., 2008; Solodkin et al., 2004). As neurons in the SMA are integrated in the functional loop controlling motor actions planning, it is therefore quite reasonable that similar preparatory aspects of the movement are engaged during MI. Kasess et al. (2008) further demonstrated that the SMA may inhibit activity of cM1 to prevent movement execution during MI. Interestingly, some authors also reported that the overlap of SMA activity during MI and motor execution is partial, and that the preSMA might rather play a specific role during imagined movements. Hence, the pattern of activity in this region could be higher during MI than during actual practice of the same task (Gerardin et al., 2000). Pre-SMA is known as being involved in acquiring new sequences, planning spatio-temporal actions, and updating motor plans for subsequent temporally ordered movements. As suggested by Malouin et al. (2003), the pre-SMA might thus be activated during MI to provide proper movement sequencing and timing. Taken together, MI would activate both the SMA and pre-SMA, not only for the preparation of the movement, but also for its suppressive influence on $\mathrm{cM} 1$ thus preventing movement execution.

Overlapping activity during MI and motor execution was also found in the premotor cortex (PMC-Guillot et al., 2008; Lotze \& Halsband, 2006; Munzert et al., 2009), with potential more robust activity in the ventral part of the PMC during MI (Gerardin et al., 2000). The dorsal part of the PMC is involved in the preparation and control of movement, while the ventral part 
may play a crucial role in the planning of movements. Neurons with mirror properties which are active during action imitation and recognition were found in this region neighbouring Brodmann areas 44/45 (Rizzolatti et al., 1998). This may partially explain why more robust activity was reported in this area during $\mathrm{MI}$ than during motor execution.

Several studies also reported that MI and motor execution activate subcortical regions such as the basal ganglia and the cerebellum (e.g., Gerardin et al., 2000; Guillot et al., 2008; Lotze \& Halsband, 2006; Munzert et al., 2009; Nair et al., 2003). These structures are known to strongly contribute to motor learning and motor preparation, and are closely connected to cortical motor-related areas. More specifically, the cerebellum contributes to predict movement outcomes as well as to correct the movement on the basis of sensori-motor feedback. Evidence that the cerebellum participates in the internal representation of movement, and therefore to MI, also comes from studies showing that cerebellar stroke disrupts MI (Battaglia et al., 2006; Gonzalez et al., 2005). In a seminal paper, Lotze et al. (1999) even postulated that the posterior cerebellum might play a crucial role in the inhibition of the motor command during MI (see also Lotze \& Halsband, 2006). Likewise, basal ganglia play a substantial role in the storage of learned motor sequences, as well as in motor preparation (Alexander \& Crutcher, 1990). While there is a general consensus regarding the activation of the basal ganglia during MI, few studies were devised to determine the specific role of this region. Li (2004) demonstrated that lesion of the putamen is likely to impair MI. More generally, however, studies on the effect of basal ganglia dysfunction on MI revealed inconsistent results (Heremans et al., 2011), with possible, but not systematic, MI impairment in patients with Parkinson's disease. Taken together, and even if experimental studies still have to understand in greater details how the cerebellum and the basal ganglia specifically contribute to the MI process, there is no doubt about their activation during imagined movements. Interestingly, distinct contributions of the corticostriatal and cortico-cerebellar anatomical systems have been proposed in the motor learning literature (Doyon \& Ungerleider, 2002; Doyon \& Benali, 2005). Although functional interactions between these anatomical systems are essential at the beginning of the learning process, there is ample evidence that the cerebellum plays a weaker role when the sequence is well learned and has reached asymptotic performance (for review, see Doyon \& Benali, 2005), while basal ganglia remain active during the later stages of motor learning. By comparing the pattern of cerebral activations in 13 skilled and 15 unskilled imagers during both physical execution and MI sequence of finger movements, Guillot et al. (2008) showed that poor imagers not only need to recruit the cortico-striatal system, but also activate the cortico-cerebellar system as well. Findings also suggested that compared to poor imagers, good imagers would have a more efficient recruitment of movement engrams. Although this remains a working hypothesis awaiting experimental investigation, the authors concluded that the pattern of cerebral activation recorded during $\mathrm{MI}$ in poor imagers might improve and evolve close to that observed in good imagers after MI training. This might suggest that the expected changes in subcortical brain activations during MI would reflect those elicited by the process of actual motor learning (see also Lafleur et al., 2002; Jackson et al., 2003). 


\subsection{Activation of parietal regions}

Activation of parietal areas including possibly the somatosensory cortex, but more certainly the inferior and superior parietal lobules and the precuneus, is frequently reported during MI (Binkofski et al., 2000; Gerardin et al., 2000; Guillot et al., 2009; Hanakawa et al., 2003; Lotze et al., 1999; Munzert et al., 2009; Nair et al., 2003). More importantly, the parietal cortex, and perhaps predominantly the left parietal cortex, could play a critical role in the formation of mental images. Experimental studies in patients with lesions located in the superior region of the parietal cortex showed that the temporal congruence between actual and MI times, known as being a reliable measure of MI quality (Guillot \& Collet, 2005; Guillot et al., 2011; Malouin et al., 2008), was affected (Malouin et al., 2004; Sabate et al., 2007; Sirigu et al., 1996). Sirigu et al. (1996) postulated that the parietal cortex might set up an internal model of the forthcoming movement making the participants to predict the expected movement outcome. After parietal damage, coding for the spatial properties of the movement to be imagined and predicting the temporal feature of the movement might therefore be more difficult, hence reflecting the critical role of the posterior parietal cortex in the use of MI.

Interestingly, bilateral parietal lesions resulted in a complete unawareness of movement execution during imagery (Schwoebel et al., 2002). In fact, the patient exhibited hand movements during the MI of the same body segments, but explicitly denied that they occurred. These data strongly support that parietal areas are critically involved in the generation and guidance of imagined movements. More importantly, they further suggest that the patient failed to inhibit the motor consequences of MI, and therefore, that parietal areas may also play a critical role in the inhibitory processes of the motor command during MI. Finally, and in conjunction with previous fMRI data (Gerardin et al., 2000), these findings suggest a functional dissociation between cortical areas underlying MI and motor execution. The primary sensory area would be more activated during motor execution, due to sensory feedback processes, whereas MI might elicit greater activity both in the inferior and superior parietal cortices, especially in the left hemisphere.

\subsection{The effect of motor imagery content}

A limited number of fMRI studies examined the influence of MI content on brain activations, and most specifically whether visual imagery and kinaesthetic imagery recruit different neural networks. In a first study looking at this issue, Binkofski et al. (2000) reported that the anterior part of the intraparietal sulcus was more active during kinaesthetic imagery of finger movements, while visual imagery yielded stronger activation in the posterior part. Bilateral activations were also reported during kinaesthetic imagery in the opercular portion of the ventral PMC. In a seminal paper, Solodkin et al. (2004) later investigated the neural networks mediating physical execution, visual imagery and kinaesthetic imagery of hand movements. Although these two types of imagery shared similar neural substrates, including the connection from the superior parietal lobule to the SMA, the main difference was found in the inputs from the superior parietal lobule and the SMA to cM1, which were opposite to those observed during motor execution. In the same line of research, Guillot et al. (2009) examined whether the same group of participants with high imagery abilities recruited similar or distinct brain 
activations during visual imagery and kinaesthetic imagery of complex hand movements. Visual imagery activated predominantly the visual pathways including the occipital regions and the precuneus, whereas the pattern of kinaesthetic imagery involved mainly motorassociated structures and the inferior parietal lobule (Figure 3). These data support the hypothesis that visual imagery of a motor sequence refers to the visual properties of visual perception, while kinaesthetic imagery includes in greater extent motor simulation processes related to the form and timing of actual movements (Michelon et al. 2006). Although visual and kinaesthetic imagery shared common brain structures, these data provide strong evidence that the patterns of neural activity mediating the ability to perform a specific MI type are partially different. Practically, it might suggest that participants are able to favor one sensory modality to form mental images, although MI is a multimodal and multidimensional construct.

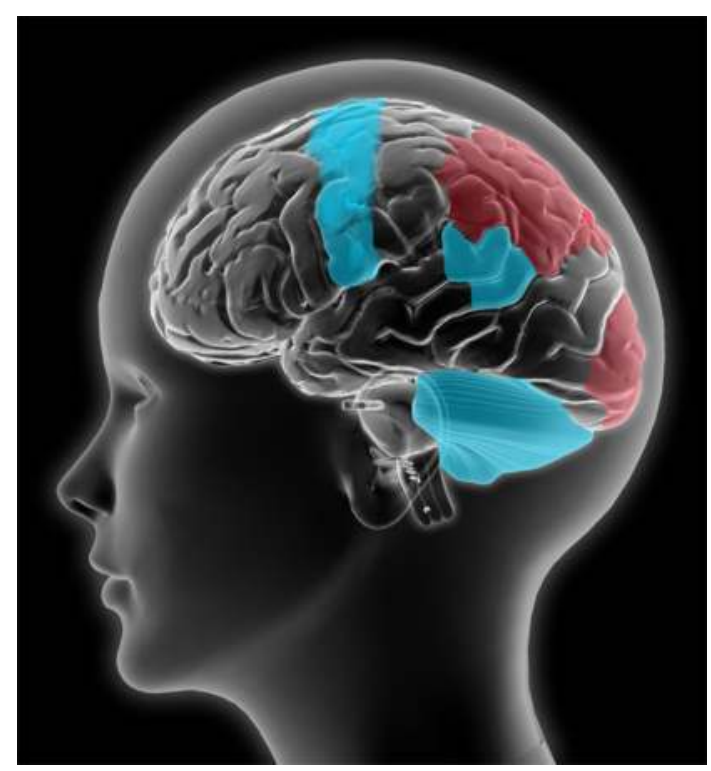

Figure 3. Schematization of brain activations during visual and kinaesthetic imagery. During kinaesthetic imagery (blue), the pattern of activity involves motor-related regions mainly, including CM1, PMC, SMA, cerebellum and basal ganglia (hidden from the view), as well as in the inferior parietal cortex. In contrast, more activity is observed during visual imagery (red) in occipital regions and superior parietal lobule, including the precuneus.

Similar data have been reported in regards to the visual imagery perspectives, that is, by comparing first and third person imagery perspectives. The first study looking at this issue, was conducted by Ruby and Decety (2001) with PET scan. They investigated the neural networks mediating MI when the participants were asked either to mentally simulate an action or to imagine someone else (i.e., the experimenter) performing the same movement. They found that the inferior parietal lobule and the somatosensory cortex were more activated during the first-person than the third-person perspective, while increased activity was observed in the precuneus and during the third-person perspective. Then, fMRI experiments 
were designed to examine in greater details whether using either the first or third-person imagery perspective lead to motor-areas-related changes. Jackson et al. (2006) explored the neural circuits involved in imitation and perspective-taking, and showed that first-person perspective was more tightly coupled to the sensory-motor system than the third-person perspective, which requires additional visuospatial transformation. More recently, Lorey et al. (2009) also found stronger activation of motor and motor-related brain structures, like the inferior parietal lobe, when imagining a movement through a first-person compared to a thirdperson perspective. They argued that proprioceptive information on actual body posture would be more relevant for internal visual imagery users, which also reveals that bodily information is mainly integrated into the image of one's own body movement.

More generally, these results provide clear evidence that both imagery types (e.g. visual vs. kinaesthetic imagery) and perspectives (first-person vs. third-person imagery) are partially mediated through separate neural systems, which may therefore contribute differently during the process of motor learning and neurological rehabilitation. This assumption is supported by a remarkable fMRI study demonstrating that MI engages systematically the organized sections of $\mathrm{cM} 1$ in a somatotopic manner, i.e., that the content of the mental images is reflected in the pattern of motor cortical activation (Ehrsson et al., 2003).

\subsection{Neural mechanisms for motor expertise}

The fact that MI efficacy depends on the individual ability to form accurate mental images is now well-established. Although not systematic, this capacity to mentally simulate forthcoming actions may be influenced by the individual level of expertise in the corresponding motor task. While the concepts of motor expertise and MI ability measurements have been considered early on, researchers are able, for a short time, to assess the content of MI objectively using thorough procedures, in particular since the advent of functional brain mapping studies (Guillot et al., 2010). Taken overall, fMRI data strongly support the existence of distinct neural mechanisms of expertise in MI, as a function of the individual skill level. For instance, lower cortical activation was recorded in professional violinists as compared to amateurs (Lotze et al., 2003). Similar differences were observed in the neural networks mediating MI in novice and expert athletes (Milton et al., 2008). By comparing brain activations of six golfers of various handicaps during MI of a golf swing, Ross et al. (2003) found decreased activations of the SMA and cerebellum as a function of golf skill level, i.e. an inverse relationship between brain activity and skill level. Also, golf swing MI yielded few peaks of activation in the basal ganglia and cingulate gyri across all skill levels. Milton et al. (2007) finally reported that the posterior cingulate, the amygdala-forebrain complex, and the basal ganglia were activated in novices but not in elite golfers during motor planning of a golf swing movement, hence confirming that the neural networks controlling both motor planning and MI are influenced by the individual skill level. As a whole, changes in cerebral activations confirmed previous investigations showing that levels of expertise during the motor learning process are supported by different neural networks (Doyon \& Benali, 2005).

Analogously, researchers investigated the neural networks mediating musical experience, training onset, and training stages. Langheim et al. (2002) first showed in participants who 
imagined playing a musical selection with their instrument that an associative network including the superior parietal lobule, the inferior frontal gyrus and the bilateral lateral cerebellum was activated during. This network would be particularly activated during the coordination of the complex spatial and timing components of musical performance. As mentioned above, Lotze et al. (2003) compared the patterns of brain activation during auditory imagery in experienced and novice musicians, with the professionals reporting frequent use of imagery with high vividness. Interestingly, experienced musicians recruited very few cerebral areas, while amateurs manifested a widely distributed activation map. In the professional group, however, more activation was observed in the SMA, the PMC, the superior parietal lobule and the cerebellum. Finally, Kleber et al. (2007) reported an increased activation in the fronto-parietal regions during imagined singing, suggesting increased involvement of working memory processes during imagery

To summarize, fMRI data provided strong evidence that partially separate neural networks are activated during MI of both motor and musical performance in regards to the individual differences in the level of expertise. These findings therefore strongly support the hypothesis of distinct neural mechanisms for expertise in imagery, independently of the imagery type, with the network integrating the superior parietal lobule being seen as mediating the imagery activity of highly experienced people.

Apart from the level of expertise itself, the rate at which the movement is performed and imagined is also likely to recruit distinct neural patterns. Accordingly, Sauvage et al. (2013) nicely demonstrated that although execution and imagination of slow and fast movements activate a common neural substrate, the rate of movement differentially yielded associative cortical, striatal and cerebellar areas. Their data suggest that slow movement and motor imagery of both slow and fast movements activated fronto-polar, orbitofrontal and dorsolateral prefrontal cortices, while fast movements recruited more intensively the premotor cortex and the cerebellum.

\section{4. fMRI advances in the study of motor imagery}

Going beyond the usual comparison of brain activation patterns that are associated with MI and motor execution of the same movement is the next step in this field of research. To do so, researchers may take advantage of the recent methodological fMRI developments. Both the use of real time fMRI and the recording of changes in functional and effective connectivity in the neural networks activated during MI are of particular interest. Also, reporting more systematically negative BOLD responses might contribute to expand our knowledge on neuronal inhibition during MI.

It is now possible to image human brain functioning in real time with fMRI (Esposito et al., 2003; Weiskopf et al., 2003; for an extensive review, see deCharms, 2008). This technique provides a reconstruction of the raw data obtained with the brain scan, while the scan is going on. There are several exciting research perspectives for considering the contribution of real time fMRI dedicated to MI study. This technique may first be useful during the MI learning 
process, especially when the pattern of activation during mental simulation is not the one expected. Indeed, real time images are expected to provide participants some objective information related to the vividness of MI, since they have been educated to gain some familiarity with the neuroanatomy before neurofeedback fMRI sessions. Receiving a feedback from brain activation in predetermined regions of interest seems possible. An average 2-5s time lapse usually remains necessary, due to the physiological delay of the haemodynamic response (a more simple feedback can also be used, e.g. a simple score using a Likert-type scale). A well-known study supporting the use of real time fMRI was published by Yoo and Jolesz (2002), who used visual feedback of brain activation to guide participants to adjust their motor task performance and to achieve the desired modulation of cortical activity. For example, during simple hand movements, participants spontaneously involved more muscle groups and increased tapping frequency along fMRI sessions. The biofeedback given to participants with regard to the activated neural network helped them to modulate their cortical activation. A significant illustration of the strength of this methodology in the field of MI was offered by deCharms et al. (2004) during imagery of a manual action task. In this study, participants received feedback about the activation level in the somatomotor cortex with a simple virtual reality interface. The results showed that they enhanced the fMRI level of activation driven by $\mathrm{MI}$ in the somatomotor cortex through the course of training. Moreover, the activation of this region after MI training was as robust as that recorded during actual practice. In a more recent study, Yoo et al. (2008) showed that real time fMRI might help individuals to learn how to increase region-specific cortical activity associated with a MI task. Practically, the level of increased activation in motor areas was consolidated after the 2-week self-practice period. Regarding the method for presentation of neurofeedback (intermittent presentation or continuous presentation) and the nature of the neurofeedback being presented to participants during a MI task (true or false neurofeedback regarding brain activations), Johnson et al. (2010) further reported that the intermittent presentation of feedback was more effective than the continuous presentation in promoting self-modulation of brain activity. Accordingly, regular interruptions in neurofeedback presentation allowed central processing and integration of the information conveyed in the feedback regarding brain activations. The authors also reported that false feedback resulted in irrelevant brain activations with regards to the regions of interest targeted by experimental instructions. Finally, Xie et al. (2011) supported the effectiveness of delivering neurofeedback during MI using real time fMRI, and further provided evidence that the SMA was controllable by participants. These data strongly support that real time fMRI is a valuable technique to investigate whether participants are able to use a cognitive strategy to control a target brain region in real time. In the field of neurorehabilitation, for example, a similar approach could be used to learn how controlling pain by learning to control the brain regions that mediate pain perception (deCharms et al., 2005). Indeed, there are multiple therapeutic applications of real time fMRI but it is too early to predict success or failure and new experimental results are awaited.

Real time fMRI might also particularly useful in developing brain-computer interfaces (deCharms, 2008). A brain-computer interface is a novel communication system that translates human thoughts or intentions into a control signal without using any muscle activity (for review, see Pfurtscheller \& Neuper, 2010). To date, many brain-computer interface systems 
have used MI tasks to modulate sensorimotor EEG activity taken to operate and control an external device. Pfurtscheller \& Neuper (2010) nicely demonstrated how brain-computer interface systems using MI can contribute to help patients with various motor impairments and paralysis. Despite the temporal limitations of fMRI, using fMRI data for brain-computer interface remains plausible as many cognitive processes change slowly, over seconds or minutes (deCharms, 2008). Practically, the method is appealing although future experimental studies are necessary to determine its feasibility and effectiveness, such as during motor recovery of patients with motor impairment. Real time fMRI might also be used to explore the state of consciousness and communicate with patients in a persistent vegetative state. Accordingly, Owen et al. (2006) detected awareness in such a patient following instructions to mentally imagine moving around a house and playing tennis. Brain activations were observed in the parahippocampal gyrus, the posterior parietal lobe and the lateral PMC during MI of walking, and in the SMA when imagining playing tennis. These data provide evidence that real time fMRI might be used along with MI to actually communicate with people and/or patient who are physically or conventionally unable to interact with their environment (deCharms, 2008).

Creating functional connectivity maps of distinct spatial distributions of temporally correlated brain regions is another methodological advanced tool offered by fMRI. Functional and effective connectivity can be used to examine interactions among brain regions. Practically, these techniques go beyond the usual activation maps obtained through peak-detection methods, by looking respectively at temporal correlation between the time course of activation of two regions, and the influence of one neuronal population over another (Doyon \& Benali, 2005; Friston \& Büchel, 2003; Friston et al., 1995). Multiple innovative data-driven methods have been proposed to investigate the changes observed in cerebral networks over time, or to study functional and effective connectivity. Some reliable examples are the structural equation modeling and the dynamic causal modeling. In the field of MI, few researchers examined the inter-relationships among brain areas selectively activated along different experimental conditions, that is, the effective connectivity between network components. Solodkin et al. (2004) explored the effective connectivity of the neural networks mediating motor execution, visual imagery, and kinaesthetic imagery of a finger to thumb opposition task. Their results provided evidence that the networks underlying these behaviors were almost different, despite the extensive overlap between motor execution and kinaesthetic imagery. In particular, inputs from SMA and lateral PMC to cM1, which were facilitated during motor execution, exhibited the opposite activity during kinaesthetic imagery, suggesting a physiological mechanism whereby the system prevents overt movements. A second study looking at the effective connectivity between SMA and cM1 suggested that the lack of activation in cM1 during MI might result from a suppressive influence of the SMA (Kasess et al., 2008). More recently, Gao et al. (2008) and Chen et al. (2009) reported that, in right-handed participants, more brain regions showed effective connections to the SMA during right-hand MI than during left-hand MI, but that the strength of the casual influence was stronger during left-hand MI. Furthermore, they found forward and backward effective connectivity between the SMA and the bilateral dorsal PMC, the contralateral primary and somatosensory cortex, and cM1. A last study by the same group of researchers confirmed these findings, and further showed that 
motor execution has some increased causal connections because of additional processes for the overt behavior stage (Gao et al., 2011). Taken together, these experimental studies highlight the advantages of studying functional and/or effective connectivity through fMRI to expand our understanding of the neural underpinnings of MI (see also Szameitat et al., 2012).

Reporting an elevation in the fMRI BOLD signal, namely positive BOLD, has become a common routine for mapping neural activity in the human brain. Aside from positive BOLD signal changes, several studies have also observed negative BOLD responses. The negative BOLD signal is a physiologic process correlated with a corresponding decrease in cerebral blood flow, oxygen consumption, and neuronal activity (for review, see Shmuel et al., 2002). Practically, negative BOLD signal reflects less neural processing for a given task as compared to a baseline condition. The negative BOLD response might be caused by a reduction in cerebral blood flow and is associated with decreases in the rate of oxygen consumption. Its neurobiological mechanisms are yet not well understood, and much of the debate has centered on whether its source is primarily vascular or neuronal. The neuronal origin might reflects a suppression of the local neuronal activity and/or a reduction in the afferent input, whereas the vascular origin refers to a reduction in cerebral blood flow to the less demanding regions due to the increase in flow to the demanding areas, without a necessary decrease in neuronal activity in the negative regions (Schmuel et al., 2002). Until recently, very few experimental studies were designed to analyze negative BOLD responses during either visual or MMI tasks. An interesting and innovative paper by Amedi et al. (2005) investigated the pattern of brain deactivation during visual imagery and compared it to the neural correlates of visual perception. While they found that visual imagery and visual perception share similar neural substrate, these two conditions yielded different brain-deactivation profiles as shown by negative BOLD responses. Of particular interest is that the authors reported a robust deactivation in early auditory areas as well as a selective deactivation in the somatosensory cortex during visual imagery, hence supporting that visual imagery is associated with deactivation of nonvisual sensory processing. Based on these data, they stated that that pure visual imagery might be characterized by an isolated activation of visual cortical areas with concurrent deactivation of sensory inputs that may potentially disrupt the image created by the mind's eye. They also considered that deactivation could be the consequence of filtering out irrelevant stimuli. The correlation between the level of deactivation and the vividness of visual imagery might support the hypothesis that participants with high imagery ability are able to shut down or disconnect the "irrelevant" cortices. To the best of our knowledge, unfortunately, there are no experimental studies specifically designed to look for similar results in MI paradigms per se. Interestingly, it has been suggested that the prolonged negative BOLD signal reflects an inhibition or suppression of neuronal firing patterns (Raichle, 1998; Kastrup et al., 2008). Looking more systematically for negative BOLD responses might therefore be of particular interest to investigate the inhibitory processes of the motor command during imagined movements. Indeed, motor command inhibition mechanisms during MI remains rather unclear since no direct fMRI brain signal is known to characterize neural inhibitory processes, and inhibition during MI has been primarily studied from brain activation signals. The negative BOLD response might therefore be an interesting methodological complement to extend our knowledge of motor inhibition 


\section{Conclusion}

The data reviewed in this chapter strongly support that recording human brain functioning with fMRI during MI provides an objective measurement of the neural networks underlying MI processes. Although they are not totally overlapping, it is now well-established that the neural substrates mediating MI and actual execution of the same task are quite similar. Spurred by recent fMRI methodological advances, the next step of MI research will certainly contribute to understand in greater details the neural correlates of imagined movements, as well as the inhibition of the motor command. MI studies could therefore benefit from the use of real time fMRI, effective connectivity, and also negative BOLD. This latter technique might be of particular interest in investigating the inhibitory processes of the motor command during MI. Cerebral regions which are inhibited during mental operations or human behavior are certainly of the same scientific interest than those which are activated.

\section{Author details}

Aymeric Guillot", Franck Di Rienzo and Christian Collet

Centre de Recherche et d'Innovation sur le Sport, Performance Motrice, Mentale et du Matériel (P3M), Université de Lyon, Université Claude Bernard Lyon1, France

\section{References}

[1] Alexander, G.E., \& Crutcher, M.D. (1990). Functional architecture of basal ganglia circuits: neural substrates of parallel processing. Trends in Neuroscience, 13, 266-271.

[2] Alkadhi, H., Brugger, P., Boendermaker, S.H., Crelier, G., Curt, A., Hepp-Reymond, M.-C., \& Kollias, S.S. (2005). What disconnection tells about motor imagery: evidence from paraplegic patients. Cerebral Cortex, 15, 131-140.

[3] Amedi, A., Malach, R., \& Pascual-Leone, A. (2005). Negative BOLD Differentiates Visual Imagery and Perception. Neuron, 48, 859-872.

[4] Battaglia, F., Quartarone, A., Ghilardi, M.F., Dattola, R., Bagnato, S., Rizzo, V., Morgante, L., \& Girlanda, P. (2006). Unilateral cerebellar stroke disrupts movement preparation and motor imagery. Clinical Neurophysiology, 117, 1009-1016.

[5] Binkofski, F., Amunts, K., Stephan, K.M., Posse, S., Schormann, T., Freund, H.J., Zilles, K., \& Seitz, R.J. (2000). Broca's region subserves imagery of motion: a combined cytoarchitectonic and fMRI study. Human Brain Mapping, 11, 273-285. 
[6] Burianová, H., Marstaller, L., Sowman, P., Tesan, G., Rich, A.N., Williams, M., Savage, G., Johnson, B.W. (2013). Multimodal functional imaging of motor imagery using a novel paradigm. NeuroImage, 71, 50-58.

[7] Chen, H., Yang, Q., Liao, W., Gong, Q., \& Shen, S. (2009). Evaluation of the effective connectivity of supplementary motor areas during motor imagery using Granger causality mapping. NeuroImage, 47, 1844-1853.

[8] Cramer, S.C., Lastra, L., Lacourse, M., \& Cohen, M.J. (2005). Brain motor system function after chronic, complete spinal cord injury. Brain, 128, 2941-2950.

[9] De Vries, S., \& Mulder, T. (2007). Motor imagery and stroke rehabilitation: a critical discussion. Journal of Rehabilitation Medicine, 39, 5-13.

[10] deCharms, R.C. (2008). Applications of real-time fMRI. Nature Review Neuroscience, 9, 720-729.

[11] deCharms, R.C., Christoff, K., Glover, G.H., Pauly, J.M., Whitfield, S., \& Gabrieli J.D. (2004). Learned regulation of spatially localized brain activation using real-time fMRI. NeuroImage, 21, 436-443.

[12] deCharms, R.C., Maeda, F., Glover, G.H., Ludlow, D., Pauly, J.M., Soneji, D., Gabrieli, J.D., \& Mackey, S.C. (2005). Control over brain activation and pain learned by using real-time functional MRI. PNAS, 102, 18626-18631.

[13] Dechent, P., Merboldt, K.D., \& Frahm, J. (2004). is the human primary motor cortex involved in motor imagery? Cognitive Brain Research, 19, 138-144.

[14] Dietrich, A. (2008). Imaging the imagination: the trouble with motor imagery. Methods, 45, 319-324.

[15] Di Rienzo, F., Guillot, A., Delpuech, C., Rode, G., Grangeon, M., \& Collet C. (2011). Inhibition of the motor command during motor imagery: a MEG study with a tetraplegic patient. $17^{\text {th }}$ Annual Meeting of the Organization on Human Brain Mapping, June 26-30, Quebec City, Canada, p. 40.

[16] Di Rienzo, F., Collet, C., Hoyek, N. \& Guillot, A. (2014a). Impact of neurologic deficits on motor imagery: A systematic review of clinical evaluations. Neuropsychology Review, 24, 116-147.

[17] Di Rienzo, F., Guillot, A., Daligault, S., Delpuech, C., Rode, G. \& Collet, C. (2014b). Motor inhibition during motor imagery: A single-case study with a quadriplegic patient. Neurocase, in press.

[18] Doyon, J., \& Benali, H. (2005). Reorganization and plasticity in the adult brain during learning of motor skills. Current Opinion in Neurobiology, 25, 161-167,

[19] Doyon, J., \& Ungerleider, L.G. (2002). Functional anatomy of motor skill learning. In: Neuropsychology of Memory, Squire L.R \& Schacter D.L. (Ed.), pp. 225-38, Guilford Press. 
[20] Driskell, J. E., Copper, C., \& Moran, A. (1994). Does mental practice enhance performance? Journal of Applied Psychology, 79, 481-492.

[21] Ehrsson, H.H., Geyer, S., \& Naito, E. (2003). Imagery of voluntary movement of fingers, toes and tongue activates corresponding body-part-specific motor representations. Journal of Neurophysiology, 90, 3304-3316.

[22] Ersland, L., Rosen, G., Lundervold, A., Smievoll, A.I., Tillung, T., Sundberg, H., \& Hugdahl, K. (1996). Phantom limb imaginary fingertapping causes primary motor cortex activation: an fMRI study. Neuroreport,8, 207-210.

[23] Esposito, F., Seifritz, E., Formisano, E., Morrone, R., Scarabino, T., Tedeschi, G., Cirillo, S., Goebel, R., \& Di Salle, F. (2003). Real-time independent component analysis of fMRI time-series. NeuroImage, 20, 2209-2224.

[24] Friston, K.J., \& Buchel, C. (2003). Functional connectivity. In: Human Brain Function, Frackowiak, R.S.J. et al., Eds), Academic Press (2 ${ }^{\text {nd }}$ Ed.).

[25] Friston, K., Ungerleider, L., Jezzard, P., \& Turner, R., (1995). Characterizing modulatory interactions between V1 and V2 in human cortex with fMRI. Human Brain Mapping, 2, 211-224.

[26] Gao, Q., Chen, H., \& Gong, Q. (2008). Evaluation of the effective connectivity of the dominant primary motor cortex during bimanual movement using Granger causality. Neuroscience Letters, 443, 1-6.

[27] Gao, Q., Duan, X., \& Chen, H. (2011). Evaluation of effective connectivity of motor areas during motor imagery and execution using conditional Granger causality. NeuroImage, 54, 1280-1288.

[28] Gerardin, E., Sirigu, A., Lehericy, S., Poline, J.B., Gaymard, B., Marsault, C., Agid, Y., \& Le Bihan, D. (2000). Partially overlapping neural networks for real and imagined hand movements. Cerebral Cortex, 10, 1093-1104.

[29] Gonzáles, B., Rodríguez, M., Ramirez, C., \& Sabaté, M. (2005). Disturbance of motor imagery after cerebellar stroke. Behavioral Neuroscience, 119, 622-626.

[30] Grezes, J., \& Decety, J. (2001). Functional anatomy of execution, mental simulation, observation, and verb generation of actions: a meta-analysis. Human Brain Mapping, 12, 1-19.

[31] Guillot, A., \& Collet, C. (2005). Duration of mentally simulated movement: a review. Journal of Motor Behavior, 37, 10-20.

[32] Guillot, A., \& Collet, C. (2008). Construction of the motor imagery integrative model in sport: a review and theoretical investigation of motor imagery use. International Review of Sport and Exercice Psychology, 1, 31-44. 
[33] Guillot, A., Collet, C., Nguyen, V.A., Malouin, F., Richards, C., \& Doyon, J. (2008). Functional neuroanatomical networks associated with expertise in motor imagery ability. NeuroImage, 41, 1471-1483.

[34] Guillot A., Collet C., Nguyen VA., Malouin F., Richards C., \& Doyon J. (2009). Brain activity during visual versus kinesthetic imagery: an fMRI study. Human Brain Mapping, 30, 2157-2172.

[35] Guillot A., Louis M., \& Collet C. (2010). Neurophysiological substrates of motor imagery ability. In: The neurophysiological foundations of mental and motor imagery, Guillot A. \& Collet C. (Eds.) pp. 109-124, Oxford University Press.

[36] Guillot, A., Hoyek, N., Louis, M., \& Collet, C. (2011). Understanding the timing of motor imagery: recent findings and future directions. International Review of Sport and Exercice Psychology, in revision.

[37] Hanakawa, T., Immisch, I., Toma, K., Dimyan, M.A., Van Gelderen, P., \& Hallett, M. (2003). Functional properties of brain areas associated with motor execution and imagery. Journal of Neurophysiology, 89, 989-1002.

[38] Hanakawa, T., Dimyan, M.A., \& Hallett, M. (2008). Motor planning, imagery, and execution in the distributed motor network: a time-course study with functional MRI. Cerebral Cortex, 18, 2775-2788.

[39] Heremans, E., Feys, P., Nieuwboer, A., Vercruysse, S., Vandenberghe, W., Sharma, N., Helsen, W. (2011). Motor imagery ability in patients with early- and mid-stage Parkinson disease. Neurorehabilitation and Neural Repair, 25, 168-177.

[40] Hétu, S., Grégoire, M., Saimpont, A., Coll, M.P., Eugène, F., Michon, P.E. \& Jackson, P.L. (2013). The neural network of motor imagery: an ALE meta-analysis. Neuroscience and Biobehavioral Reviews, 37-930-949.

[41] Holmes, P.S., \& Collins, D.J. (2001). The PETTLEP approach to motor imagery: A functional equivalence model for sport psychologists. Journal of Applied Sport Psychology, 13, 60-83.

[42] Jackson, P.L., Meltzoff, A.L., \& Decety, J. (2006). Neural circuits involved in imitation and perspective-taking. NeuroImage, 31, 429-439.

[43] Jackson, P.L., Lafleur, M.F., Malouin, F., Richards, C.L., \& Doyon, J. (2003). Functional cerebral reorganization following motor sequence learning through mental practice with motor imagery. NeuroImage, 20, 1171-1180.

[44] Jeannerod, M. (1994). The representing brain: neural correlates of motor intention and imagery. Behavioral and Brain Sciences, 17, 187-202, 1994.

[45] Johnson, S.H., Rotte,M., Grafton, S.T., Hinrichs, H., Gazzaniga,M.S., \& Heinze, H.J. (2002). Selective activation of a parietofrontal circuit during implicitly imagined prehension. NeuroImage, 17, 1693-1704. 
[46] Johnson, K.A., Hartwell, K., Lematty, T., Borckardt, J., Morgan, P.S., Govindarajan, K., Brady, K., \& George, M.S. (2010). Intermittent "Real-time" fMRI Feedback Is Superior to Continuous Presentation for a Motor Imagery Task: A Pilot Study. Journal of Neuroimaging, in press, doi: 10.1111/j.1552-6569.2010.00529.

[47] Kasess, C.H., Windischberger, C., Cunnington, R., Lanzenberger, R., Pezawas, L., \& Moser, E. (2008). The suppressive influence of SMA on M1 in motor imagery revealed by fMRI and dynamic causal modeling. NeuroImage, 40, 828-837.

[48] Kastrup, A., Baudewig, J., Schnaudigel, S., Huonker, R., Becker, L., Sohns, J.M., Dechent, P., Klingner, C., \& Wittec, O.W. (2008). Behavioral correlates of negative BOLD signal changes in the primary somatosensory cortex. Neuroimage, 41, 1341-1371.

[49] Kleber, B., Birbaumer, N., Veit, R., Trevorrow, T., \& Lotze, M. (2007). Overt and imagined singing of an Italian aria. NeuroImage, 36, 889-900.

[50] Kosslyn, S.M., Segar, C., Pani, J., \& Hillger, L.A. (1990). When is imagery used in everyday life? A diary study. Journal of Mental Imagery, 14, 131-52.

[51] Kuhtz-Buschbeck, J.P., Mahnkopf, C., Holzknecht, C., Siebner, H., Ulmer, S., \& Jansen, O. (2003). Effector-independent representations of simple and complex imagined finger movements: a combined fMRI and TMS study. European Journal of Neuroscience, $18,3375-3387$.

[52] Lafleur, M.F., Jackson, P.L., Malouin, F., Richards, C.L., Evans, A.C., \& Doyon, J. (2002). Motor learning produces parallel dynamic functional changes during the execution and imagination of sequential foot movements. NeuroImage, 2, 142-57.

[53] Langheim, F.J.P., Callicott, J.H., Mattay, V.S., Duyn, J.H., \& Weinberger, D.R. (2002). Cortical systems associated with covert music rehearsal. NeuroImage, 16, 901-908, 2002.

[54] Leonardo, M., Fieldman, J., Sadato, N., Campbell, G., Ibanez, V., Cohen, L., Deiber, M.-P., Jezzard, P., Pons, T., Turner, R., Le Bihan, D., \& Hallett, M. (1995). A functional magnetic resonance imaging study of cortical regions associated with motor task execution and motor ideation in humans. Human Brain Mapping, 3, 83-92.

[55] Li, C.R. (2000). Impairment of motor imagery in putamen lesions in humans. Neuroscience Letters, 287, 13-16.

[56] Logothetis, N. K. (2008). What we can do and what we cannot do with fMRI. Nature, $453,869-878$.

[57] Lorey, B., Bischoff, M., Pilgramm, S., Stark, R., Munzert, J., \& Zentgraf, K. (2009). The embodied nature of motor imagery: the influence of posture and perspective. Experimental Brain Research, 194, 233-243.

[58] Lotze, M., \& Halsband, U. (2006). Motor imagery. Journal of Physiology (Paris), 99, 386-395. 
[59] Lotze, L., Montoya, P., Erb, M., Hulsmann, E., Flor, H., Klause, U., Birbaumer, N., \& Grodd, W. (1999). Activation of cortical and cerebellar motor areas during executed and imagined hand movements: an fMRI study. Journal of Cognitive Neuroscience, 11, 491-501.

[60] Lotze, M., Scheler, G., Tan, H.R.M., Braun, C., \& Birbaumer, N. (2003). The musician's brain: functional imaging of amateurs and professionals during performance and imagery. NeuroImage, 20, 1817-1829, 2003.

[61] Lotze, M. \& Zentgraf, K. (2010). Contribution of the primary motor cortex to motor imagery. In: The neurophysiological foundations of mental and motor imagery, Guillot A. \& Collet C. (Eds.), pp. 31-46, Oxford University Press.

[62] Macuga, K.L., \& Frey, S.H. (2011). Neural representations involved in observed, imagined, and imitated actions are dissociable and hierarchically organized. NeuroImage, in press.

[63] Malouin, F., Belleville, S., Richards, C. L., Desrosiers, J., \& Doyon, J. (2004). Working memory and mental practice outcomes after stroke. Archives of Physical Medicine and Rehabilitation, 85, 177-183.

[64] Malouin, F., Richards, C., Durand, A., \& Doyon, J. (2008). Reliability of mental chronometry for assessing motor imagery ability after stroke. Archives of Physical Medicine and Rehabilitation, 89, 311-319.

[65] Malouin, F., Richards, C.L., Jackson, P.L., Dumas, F., \& Doyon, J. (2003). Brain activations during motor imagery of locomotor-related tasks: a PET study. Human Brain Mapping, 19, 47-62.

[66] Marx, E., Deutschländer, A., Stephan, T., Dieterich, M., Wiesmann, M., \& Brandt, T. (2004). Eyes open and eyes closed as rest conditions: Impact on brain activation patterns. NeuroImage, 21, 1818-1824.

[67] Marx, E., Stephan, T., Nolte, A., Deutschländer, A., Seelos, K.C., Dieterich, M., \& Brandt, T. (2003). Eye closure in darkness animates sensory systems. Neurolmage, 19, 924-934.

[68] Michelon, P., Vettel, J.M., \& Zacks, J.M. (2006). Lateral somatotopic organization during imagined and prepared movements. Journal of Neurophysiology, 95, 811-822.

[69] Milton, J., Solodkin, A., Hlustîk, P., \& Small, S.L. (2007).The mind of expert motor performance is cool and focused. NeuroImage, 35, 804-813.

[70] Milton, J., Small, S.L., \& Solodkin, A. (2008). Imaging motor imagery: methodological issues related to expertise. Methods, 45, 336-341.

[71] Munzert, J., Lorey, B., \& Zentraf, K. (2009). Cognitive motor processes: The role of motor imagery in the study of motor representations. Brain Research Reviews, 60, 306-326. 
[72] Murphy, S., Nordin, S.M., \& Cumming, J. (2008). Imagery in sport, exercise and dance. In: Advances in sport psychology, Horn T.S. (Eds.), pp. 306-315, Champagne, IL: Human Kinetics.

[73] Nair, D.G., Purcott, K.L., Fuchs, A., Steinberg, F., \& Kelso, J.A.K. (2003). Cortical and cerebellar activity of the human brain during imagined and executed unimanual and bimanual action sequences: a functional MRI study. Cognitive Brain Research, 15, 250-260.

[74] Olsson, C.J., Jonsson, B., \& Nyberg, L. (2008). Internal imagery training in active high jumpers. Scandinavian Journal of Psychology, 49, 133-140.

[75] Owen, A.M., Coleman, M.R., Boly, M., Davis, M.H., Laureys, S., \& Pickard, J.D. (2006). Detecting Awareness in the Vegetative State. Science, 313, 1402.

[76] Pfurtscheller, G., \& Neuper, K. (2010). EEG-based brain-computer communication. In: The neurophysiological foundations of mental and motor imagery, Guillot A. \& Collet C. (Eds.), pp. 203-212, Oxford University Press.

[77] Porro, C.A., Francescato, M.P., Cettolo, V., Diamond, M.E., Baraldi, P., Zuiani, C., Bazzocchi, M., \& Di Prampero, P.E. (1996). Primary motor and sensory cortex activation during motor performance and motor imagery: a functional magnetic resonance imaging study. Journal of Neuroscience, 16, 7688-7698.

[78] Porro, C.A., Cettolo, V., Francescato, M.P., \& Baraldi, P. (2000). Ipsilateral involvement of primary motor cortex during motor imagery. European Journal of Neuroscience, 12, 3059-3063.

[79] Raichle, M.E. (1998). Behind the scenes of functional brain imaging: a historical and physiological perspective. Proceedings of the National Academy of Sciences (USA), 95, 765-772.

[80] Rizzolatti, G., Luppino, G., \& Matelli, M. (1998). The organization of the cortical motor system: new concepts. Electroencephalographic and Clinical Neurophysiology, 106, 283-296.

[81] Roland, P.E., Larsen, B., Lassen, N.A., \& Skinhoj, E. (1980). Supplementary motor area and other cortical areas in organisation of voluntary movements in man. Journal of Neurophysiology, 43,118-136.

[82] Ross, J.S., Tkach, J., Ruggieri, P.M., Lieber, M., \& Lapresto, E. (2003). The mind's eye: Functional MR imaging of golf motor imagery. American Journal of Neuroradiology, 24, 1036-1044.

[83] Roth, M., Decety, J., Raybaudi, M., Massarelli, R., Delon-Martin, C., Segebarth, C.M., Morand, S., Gemignani, A., Decorps, M., \& Jeannerod, M. (1996). Possible involvement of primary motor cortex in mentally simulated movement: A functional magnetic resonance imagery study. Neuroreport, 7, 1280-1284. 
[84] Ruby, P., \& Decety, J. (2001). Effect of subjective perspective taking during simulation of action: a PET investigation of agency. Nature Neuroscience, 4, 546-50.

[85] Sabate, M., Gonzalez, B., \& Rodriguez, M. (2007). Adapting movement planning to motor impairments: The motor-scanning system. Neuropsychologia, 45, 378-386.

[86] Sabbah, P., Simond, G., Levrier, O., Habib M, Trabaud V, Murayama N, Mazoyer BM, Briant JF, Raybaud C, \& Salamon G. (1995). Functional magnetic resonance imaging at $1.5 \mathrm{~T}$ during sensory motor and cognitive tasks. European Neurology, 35, 131-135.

[87] Sanes, J.N., \& Donoghue, J.P. (2000). Plasticity and primary motor cortex. Annual Review of Neuroscience, 23, 393-415.

[88] Sauvage, C., Jissendi, P., Seignan S., Manto, M. \& Habas, C. (2013). Brain areas involved in the control of speed during a motor sequence of the foot: Real movement versus mental imagery. Journaol of Neuroradiology, 40, 267-280.

[89] Schmuel, A., Yacoub, E., Pfeuffer, J., Van de Moortele, P.F., Adriany, G., Hu, X., \& Ugurbil, K. (2002). Sustained negative BOLD, blood flow and oxygen consumption response and its coupling to the positive response in the human brain. Neuron, 36, 1195-1210.

[90] Schwoebel, J., Boronat, C.B., \& Coslett, H.B. (2002). The man who executed "imagined" movements: evidence for dissociable components of the body schema. Brain and Cognition, 50, 1-16.

[91] Sharma, M., Pomeroy, V.M., \& Baron, J.C. (2006). Motor imagery: a backdoor to the motor system after stroke? Stroke, 37, 1941-1952.

[92] Sharma, M., Jones, P.S., Carpenter, T.A., \& Baron, J.C. (2008). Mapping the involvement of BA $4 \mathrm{a}$ and $4 \mathrm{p}$ during motor imagery. NeuroImage, 41, 92-99.

[93] Sirigu, A., Duhamel, J.R., Cohen, L., Pillon, B., Dubois, B., \& Agid, Y. (1996). The mental representation of hand movements after parietal cortex damage. Science, 273, 1564-1568.

[94] Solodkin, A., Hlustik, P., Chen, E.E., \& Small, S.L. (2004). Fine modulation in network activation during motor execution and motor imagery. Cerebral Cortex, 14, 1246-1255.

[95] Stinear, C.M. (2010). Corticospinal facilitation during motor imagery. In: The neurophysiological foundations of mental and motor imagery, Guillot A. \& Collet C. (Eds.), pp. 47-61, Oxford University Press.

[96] Szameitat, A.J., McNamara, A., Shen, S. \& Sterr, A. (2012). Neural activation and functional connectivity during motor imagery of bimanual everyday actions. PLoS One, 7, e38506.

[97] Weiskopf, N., Veit, R., Erb, M., Mathiak, K., Grodd, W., Goebel, R., \& Birbaumer, N. (2003). Physiological self-regulation of regional brain activity using real-time func- 
tional magnetic resonance imaging (fMRI): methodology and exemplary data. NeuroImage, 19, 577-86.

[98] Xie, B., Ma, X., Yao, L., Long, Z., \& Zhao, X. (2011). Real time fMRI data analysis using region of interest selection based on fast ICA. Progress in Biomedical Optics and Imaging - Proceedings of SPIE, 7965, article 79651s.

[99] Yoo, S.S, \& Jolesz, F.A. (2002). Functional MRI for neurofeedback: feasability study on a hand motor task. Neuroreport, 13, 1377-81.

[100] Yoo, S.S., Lee, J.H., O’Leary, H., Panych, L.P., \& Jolesz, F.A. (2008). Neurofeedback fMRI-mediated learning and consolidation of regional brain activation during motor imagery. International Journal of Imaging Systems and Tecnhology, 18, 69-78.

[101] Zang, Y., Jia, F., Weng, X., Li, E., Cui, S., Wang, Y., Hazeltine, E., \& Ivry, R. (2003). Functional organization of the primary motor cortex characterized by event-related fMRI during movement preparation and execution. Neuroscience Letters, 337, 69-72. 